\title{
Second-harmonic generation via double topological valley-Hall kink modes in all-dielectric photonic crystals
}

\author{
Zhihao Lan $\odot,{ }^{1}$ Jian Wei You $\odot,{ }^{1}$ Qun Ren $\odot,{ }^{1,2}$ Wei E. I. Sha, ${ }^{1,3}$ and Nicolae C. Panoiu ${ }^{1}$ \\ ${ }^{1}$ Department of Electronic and Electrical Engineering, University College London, Torrington Place, London WC1E 7JE, United Kingdom \\ ${ }^{2}$ School of Electrical and Information Engineering, Tianjin University, Tianjin 300072, China \\ ${ }^{3}$ State Key Laboratory of Modern Optical Instrumentation, College of Information Science and Electronic Engineering, \\ Zhejiang University, Hangzhou 310027, China
}

(Received 8 July 2020; accepted 29 March 2021; published 21 April 2021)

\begin{abstract}
Nonlinear topological photonics, which explores topics common to the fields of topological phases and nonlinear optics, is expected to open up a new paradigm in topological photonics. Here, we demonstrate second-harmonic generation (SHG) via nonlinear interaction of double topological valley-Hall kink modes in all-dielectric photonic crystals ( $\mathrm{PhCs}$ ). We first show that two topological frequency band gaps can be created around a pair of frequencies, $\omega_{0}$ and $2 \omega_{0}$, by gapping out the corresponding Dirac points in two-dimensional honeycomb PhCs. Valley-Hall kink modes along a kink-type domain wall interface between two PhCs placed together in a mirror-symmetric manner are generated within the two frequency band gaps. Importantly, through full-wave simulations and mode dispersion analysis, we demonstrate that tunable, bidirectional phase-matched SHG via nonlinear interaction of the valley-Hall kink modes inside the two band gaps can be achieved. In particular, by using Stokes parameters associated with the magnetic part of the valley-Hall kink modes, we introduce the concept of SHG directional dichroism, which is employed to characterize optical probes for sensing chiral molecules. Our work opens up avenues toward topologically protected nonlinear frequency mixing and active photonic devices implemented in all-dielectric material platforms.
\end{abstract}

DOI: 10.1103/PhysRevA.103.L041502

Introduction. Recent advances in topological photonics [1-7] have led to new ways to control light in a robust manner using photonic states that are protected by the topological properties of the systems. Earlier works [8-11] in this field have focused on the realization of the photonic analog of quantum Hall states in two-dimensional (2D) photonic crystals (PhCs) containing magneto-optical materials, where the time-reversal symmetry is broken by external magnetic fields. As magneto-optical effects are generally weak at optical frequencies, intense research efforts were devoted to topological photonic systems without magneto-optical materials, and concepts such as Floquet topological phases and synthetic magnetic fields have been demonstrated in helical waveguide arrays [12] and coupled-ring resonators [13], respectively. Furthermore, photonic systems emulating quantum spin Hall [14-17] and quantum valley Hall [18-21] effects, which preserve the time-reversal symmetry of the system, have also been proposed. We note that for all systems discussed above, the topological photonic properties can be understood within the single-particle framework.

On the other hand, interacting topological phases provide an exciting topic in condensed-matter physics [22], and in the context of photonics, the existence of nonlinearity in many optical materials [23] provides a unique platform to study interaction effects in topological physics, which is expected to greatly expand our understanding of topological photonic systems [24]. Indeed, lattice edge solitons [25-27], nonlinear control [28] and imaging [29] of photonic topological edge states, traveling-wave amplifiers [30], topological insulator lasers [31,32], topological sources of quantum light [33], and the potential to enhance harmonic generation [34-36] have been demonstrated. Despite these advances in understanding the nonlinear effects in topological photonic systems, achieving nonlinear frequency mixing - one of the fundamental nonlinear optical processes_-via phase matching topological edge states is still largely unexplored. Recently, we studied four-wave mixing of topological edge plasmons in graphene metasurfaces [37] and second- (SHG) and third-harmonic generation in topological PhCs [38] using one-way edge modes similar to quantum Hall states with external magnetic field. Nevertheless, whether the above goal can be realized in time-reversal symmetry-preserving topological photonic systems without exploiting magneto-optical effects is still an open question, which we address here.

In this Letter, we demonstrate SHG in all-dielectric PhCs, through nonlinear interaction of topological edge modes within two different frequency band gaps around $\omega_{0}$ and $2 \omega_{0}$. Our implementation is based on the photonic quantum valley Hall effect and its associated valley-Hall kink modes [18-21]. A key result of our work lies in the design of two topological valley gaps hosting double valley-Hall kink modes that can be phase matched to achieve SHG. Importantly, unlike the case of one-way edge modes [37,38], in the current system one could launch the fundamental wave along either direction of the topological interface, thanks to the topology of the valley-Hall kink modes, with second-harmonic waves being 

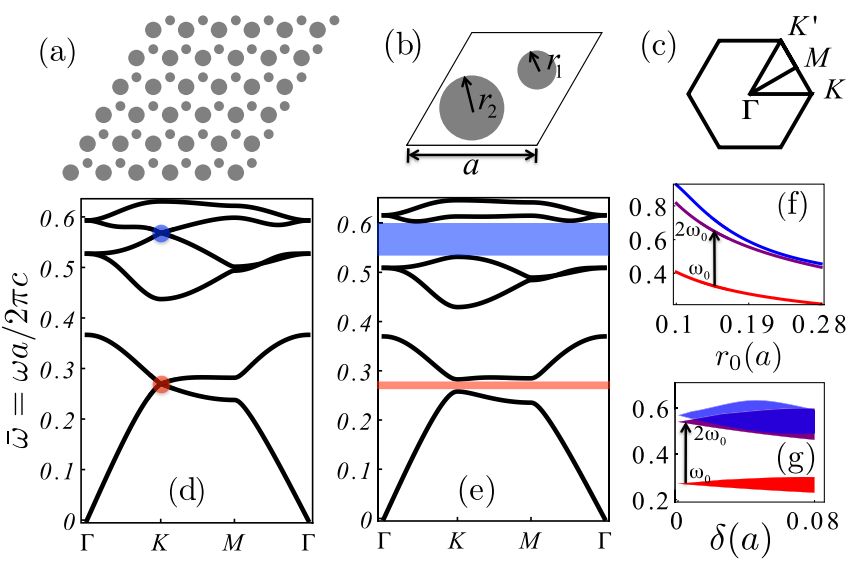

FIG. 1. Emergence of two valley gaps for SHG. (a) Schematic of the system. (b) Unit cell of the PhC, containing two cylinders of nonlinear material with radii $r_{1}$ and $r_{2}$, dielectric constant $\epsilon=12$, and $\chi^{(2)}=10^{-21} \mathrm{CV}^{-2}$. (c) First Brillouin zone of the PhC. (d) The existence of double Dirac points (marked by red and blue dots) of the $\mathrm{PhC}$ at $r_{1}=r_{2}=0.2 a$. (e) The same as in (d), but calculated for $r_{1}=$ $0.18 a$ and $r_{2}=0.22 a$. (f) The frequencies of the two Dirac points in (d) vs $r_{0}$ when $r_{1}=r_{2}=r_{0}$. (g) The width of the two valley gaps in (e) vs $\delta$, defined as $r_{1}=r_{0}-\delta$ and $r_{2}=r_{0}+\delta$ with $r_{0}=0.2 a$.

generated both in the forward and backward directions due to the time-reversal symmetry of the system. Moreover, a unique feature of our system relevant to many applications is that the amplitudes of these two wave components can be readily tuned by varying the frequency or source location and chirality, a functionality that nontopological nonlinear optics cannot provide.

The system. To demonstrate the main ideas, we consider a 2D honeycomb $\mathrm{PhC}$ made of dielectric cylinders with radii $r_{1}$ and $r_{2}$ [see Fig. 1(a)], whose unit cell and first Brillouin zone are shown in Figs. 1(b) and 1(c), respectively. The cylinders are made of nonlinear material with dielectric constant $\epsilon$ and second-order nonlinear susceptibility $\chi^{(2)}$. In the following, we use normalized frequency and momentum, $\bar{\omega}=\omega a / 2 \pi c$ and $\bar{k}=k a / \pi$, with $c$ being the speed of light and $a$ being the lattice constant. The transverse-magnetic (TM) modes of the honeycomb PhC possess Dirac points between the first and second bands $[39,40]$, a feature exploited for topological valley transport. However, Dirac points at higher bands have been less studied. We show in Fig. 1(d) the first six TM bands, determined using BANDSOLVE [41], when $r_{1}=r_{2} \equiv r_{0}$, from which one can see the existence of double Dirac points, whose frequencies as a function of $r_{0}$ are shown in Fig. 1(f).

The Dirac points could be gapped out by using a unit cell containing cylinders with different radii. We show in Fig. 1(e) the band structure of the PhC with $r_{1}=0.18 a$ and $r_{2}=0.22 a$, from which one can see the gapping out of the Dirac points by forming valley gaps. The effect of the inversion symmetry breaking can be quantified by $\left|r_{2}-r_{1}\right|$. We further show in Fig. 1(g) the width of the two valley gaps when varying the radius difference. As can be seen, the second-harmonic gap [purple (medium gray)] with respect to the first valley gap [red (light gray)] overlaps significantly with the second valley gap [blue (dark gray)]; thus, the two valley gaps [red (light gray) and blue (dark gray)] can be used for SHG. (a)

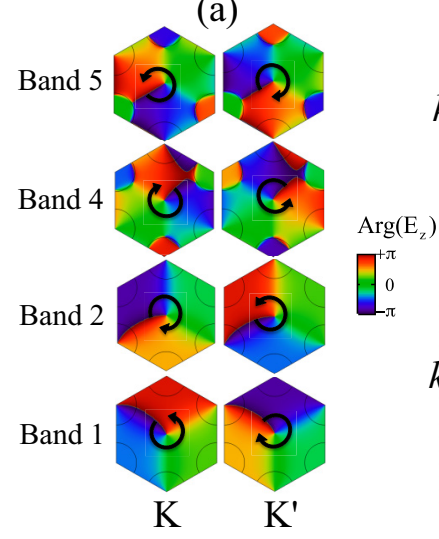

(b)

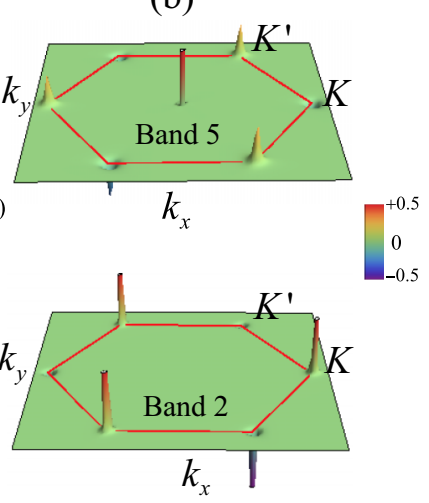

FIG. 2. Topological properties of the two valley gaps. (a) Phase winding behaviors of $E_{z}$ at $K$ and $K^{\prime}$ for the four bands around the Dirac points in Fig. 1(d). (b) Berry curvature distributions of bands 2 and 5 (Berry curvatures of bands 1 and 4 around $K, K^{\prime}$ show behaviors opposite to bands 2 and 5 and thus are not shown). The peak of Berry curvature at the $\Gamma$ point of band 5 is due to the band degeneracy between bands 5 and 6 at $\Gamma$. In the simulations, a small cylinder difference is used to gap out the Dirac points, and we have checked the integral of the Berry curvature around $K$ and $K^{\prime}$ gives $\pm \pi$.

Topological properties of the valley gaps. To demonstrate the topological nature of the two valley gaps, we present in Fig. 2 the phase-winding behavior of $E_{z}$ and the Berry curvature distribution around the two valleys, $K$ and $K^{\prime}$ [42-44]. Figure 2(a) shows that the phase-winding behavior of $E_{z}$ for the first valley gap between bands 1 and 2 is opposite to that of the second valley gap between bands 4 and 5 . Moreover, they are opposite to each other at the $K$ and $K^{\prime}$ valleys for all four bands.

The band topology is characterized by the Berry curvature $\mathcal{F}(\mathbf{k})=\nabla_{\mathbf{k}} \times \mathcal{A}_{n}(\mathbf{k})$, where $\mathcal{A}_{n}(\mathbf{k})=\left\langle u_{n \mathbf{k}}\left|i \nabla_{\mathbf{k}}\right| u_{n \mathbf{k}}\right\rangle$ is the Berry connection, with $\left|u_{n \mathbf{k}}\right\rangle$ being the eigenmode of the $n$th band. The integral of the Berry curvature over the first Brillouin zone modulo $2 \pi$ gives the Chern number, and this integral is zero for systems with time-reversal symmetry [9]. However, the Berry curvature can have nontrivial local distributions around $K$ and $K^{\prime}$, which can be used to define the valley Chern number $( \pm 1 / 2)$. From Fig. 2(b), we can see that the Berry curvature distributions have opposite signs at $K$ and $K^{\prime}$ and are opposite for the first and second valley gaps, too. As we will show later on, these features have important implications on the kink modes induced within the two gaps around $K$ and $K^{\prime}$.

Emergence of chiral valley-Hall kink modes within the two valley gaps. According to the bulk-edge correspondence principle, for an interface separating two bulk systems, if the difference in the topological invariants of the bulk systems across the interface is nontrivial, interface states will emerge inside the bulk band gap. For our case, if one $\mathrm{PhC}$ (I) is inversion symmetric to the other (II), the two valleys will be transformed into each other, i.e., $K_{I} / K_{I}^{\prime}=K_{I I}^{\prime} / K_{I I}$. As the valley Chern number at $K, K^{\prime}$ is $\pm 1 / 2$ [see Fig. 2(b)], $C_{K / K^{\prime}}^{I}=-C_{K / K^{\prime}}^{I I}= \pm 1 / 2$, and consequently, $C_{K / K^{\prime}}^{I}-C_{K / K^{\prime}}^{I I}=$ \pm 1 . This means that at one valley there exists an interface 

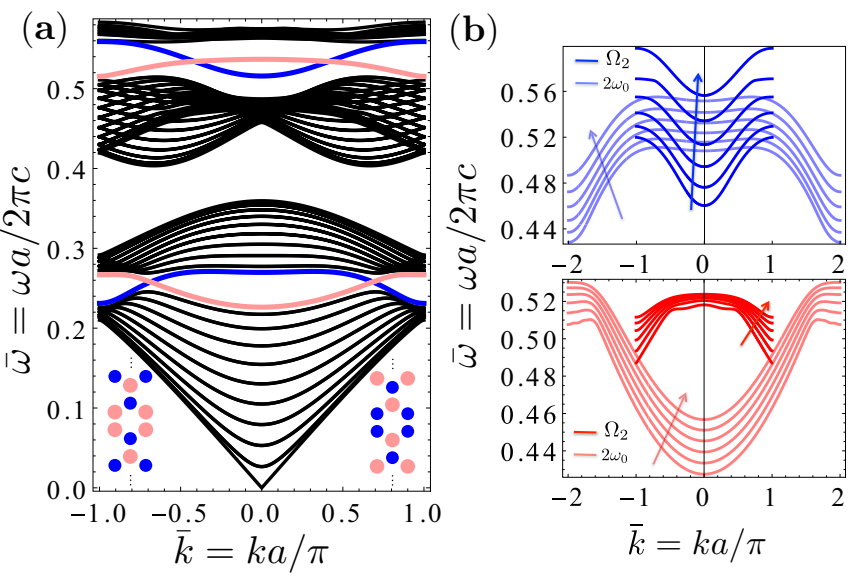

FIG. 3. Emergence of valley-Hall kink modes in the two valley gaps for SHG. (a) Band structure of a kink-type domain-wall interface separating two $\mathrm{PhCs}$ (with $r_{1}=0.2 a, r_{2}=0.23 a$ ) that are mirror symmetric to each other (see the insets). Note the red (light gray) and blue (dark gray) kink modes correspond to the interface with bigger cylinders (red, left inset) and the interface with smaller cylinders (blue, right inset), respectively. (b) Tuning the kink mode dispersion to achieve phase matching for SHG, where the fundamental modes $\omega_{0}$ are shown in the second-harmonic gap by applying the transformation $\left(\omega_{0}, k_{f}\right) \mapsto\left(2 \omega_{0}, 2 k_{f}\right)$ and $\Omega_{2}$ refers to the secondharmonic modes. Along the arrow direction, $r_{1}$ varies from $0.23 a$ to $0.18 a$ with a step of $0.01 a$, whereas $r_{2}=0.24 a$.

mode with positive group velocity $(\mathrm{GV})$, and another one with negative GV exists at the other valley. Importantly, as the two valley gaps have opposite topologies (see Fig. 2), the interface modes within the two valley gaps at the same valley have opposite GVs, a unique feature for achieving phase matching. The band structure of a kink-type domain-wall interface is calculated and presented in Fig. 3(a). One can see that the kink modes within the two valley gaps indeed have opposite GVs (slopes) at a specific valley (for further topological properties of the valley modes, see [45]).

To tailor the kink modes for efficient SHG, we show in Fig. 3(b) the evolution of the kink mode dispersion curves when changing the radius difference of $r_{1}$ and $r_{2}$. When increasing $\left|r_{1}-r_{2}\right|$, as indicated by the arrow direction, the widths of the two valley gaps increase, and the dispersion curves move toward higher frequencies. As we require the band gaps to be relatively large, one could expect to achieve efficient SHG at a large radius difference. From Fig. 3(b), we can see that when $\left|r_{1}-r_{2}\right|$ is large, the dispersion curves of the fundamental and second-harmonic kink modes corresponding to the smaller cylinder interface (top panel) move away from each other; thus, phase matching cannot be realized effectively in this case. Conversely, the dispersion curves corresponding to the larger cylinder interface (bottom panel) are well overlapped for a large radius difference, which is ideal for fulfilling phase-matching requirements. Importantly, Fig. 3(b) suggests that near the $\Gamma$-point it is possible to achieve phase matching when both the fundamental and second-harmonic waves are in the slow-light regime $\left(v_{g} \ll c\right)$, which results in greatly enhanced SHG.
SHG via double valley-Hall kink modes. For clear illustration of the SHG, we present both the fundamental and second-harmonic kink modes in the second valley gap [see Fig. 4(a)]. Due to the time-reversal symmetry of our system, at each frequency there are two kink modes, corresponding to the two valleys at $K$ and $K^{\prime}$, for both the fundamental and the second-harmonic components. Although one can excite the fundamental wave unidirectionally exploiting the inherent chirality of the kink modes, e.g., by using sources of either right- or left-circularly polarized light, generally, the generated second-harmonic waves will have both forward- and backward-propagating components.

The intrinsic, local chirality of the kink modes can be characterized by Stokes parameters defined for the magnetic field $\mathbf{H}=\left(H_{x}, H_{y}\right)$ as $D=S_{3} / S_{0}$, with $S_{0}=\left|H_{x}\right|^{2}+\left|H_{y}\right|^{2}$ and $S_{3}=-2 \operatorname{Im}\left(H_{x} H_{y}^{*}\right)$ (for details, see [45]). The chirality maps for both the fundamental and harmonic waves at the marked points of $\bar{k}_{f}$ and $\bar{k}_{s}^{+}$in Fig. 4(a) are shown in the right panels. Guided by this map, we can see that kink modes can be excited unidirectionally, with an example being given in Fig. 4(b), where the three kink modes participating in the SHG process and the frequency are indicated in Fig. 4(a) by dots and the blue (dark gray) line, respectively (see [45] for further details). While the fundamental wave at $\bar{k}_{f}$ is launched unidirectionally rightward (the source is marked by the arrow) [46,47], from the field of $\left|E_{2}\right|$, one can see that, indeed, both forward- and backward-propagating waves are generated.

To analyze the SHG process quantitatively, we perform the Fourier transform of fields $E_{1}$ and $E_{2}$ in Fig. 4(b) and present the results in Figs. 4(c) and 4(d). In particular, the peak of $\left|\tilde{E}_{1}^{k}\right|$ corresponds to $\bar{k}_{f}$ as the fundamental wave is excited unidirectionally. Interestingly, we can see three peaks in the spectrum of $\left|\tilde{E}_{2}^{k}\right|$, two of which correspond to $\bar{k}_{s}^{-}$and $\bar{k}_{s}^{+}$; that is, the forward- and backward-propagating waves at the second harmonic are generated due to their (nearly phase matched) nonlinear interaction with the fundamental wave at $\bar{k}_{f}$. The peak at $2 \bar{k}_{f}-2$, on the other hand, corresponds to a nonlinear umklapp process. It is due solely to the nonlinear polarization $P_{2} \sim \chi^{(2)} E_{1}^{2}$, and since it is not a phase-matched process, the corresponding SHG does not grow exponentially. From the amplitudes of the peaks located at $\bar{k}_{s}^{-}$and $\bar{k}_{s}^{+}$, we can observe that the forward component with $\bar{k}_{s}^{-}$is much larger due to a smaller wave-vector mismatch; the corresponding forward-to-backward ratio, $\eta_{\rightleftarrows}=\eta_{\rightarrow} / \eta_{\leftarrow}$, of the generated second-harmonic waves as a function of frequency is show in Fig. 4(e). This analysis also reveals a key feature of the SHG process, i.e., that $\eta \rightleftarrows$ can be varied simply by tuning the source frequency, a functionality that nontopological nonlinear optics does not provide. We have also verified that the period $\lambda_{p}$ in Fig. 4(b) does relate to $\bar{k}_{f}$ and $\bar{k}_{s}^{-}$via $\lambda_{p}=2 \pi /|\Delta \bar{k}|$, with $\Delta \bar{k}=\left(2 \bar{k}_{f}-2\right)-\bar{k}_{s}^{-}$, meaning that the observed oscillations are due to the beating between the two rightward-generated waves.

To further confirm that the waves participating in the SHG process corresponding to the full-wave simulations presented in Fig. 4(b) are, indeed, those suggested by the eigenmode calculations, we scan the excitation frequency [blue (dark gray) line in Fig. 4(a)] from the bottom of the frequency-matching window to its top and present in Fig. 4(f) the extracted kink mode dispersions together with those shown in Fig. 4(a). The 

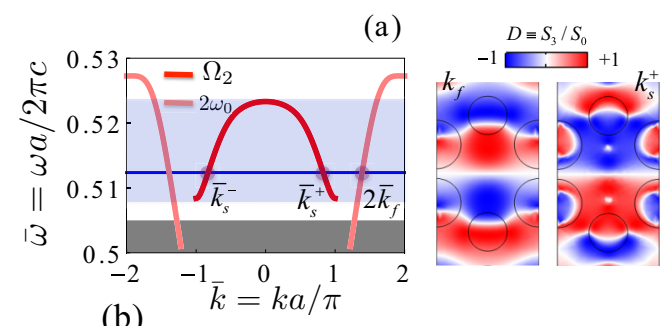

(b)

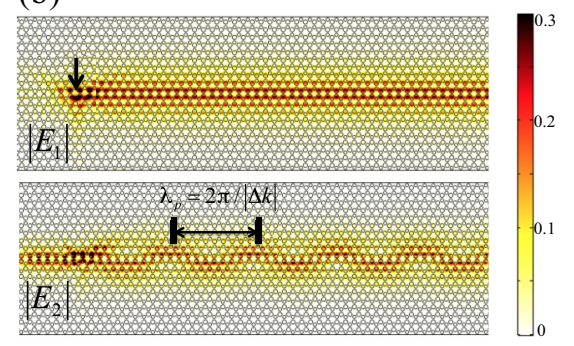

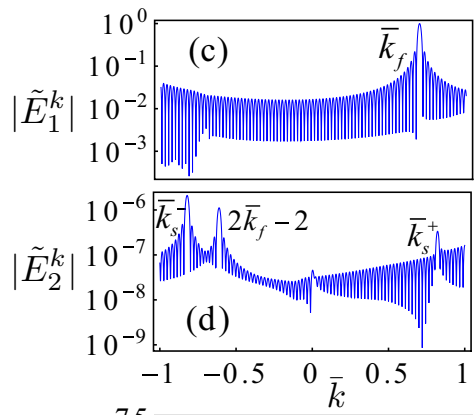

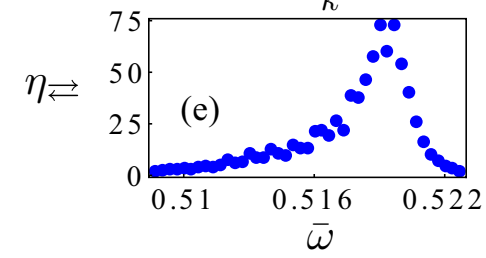

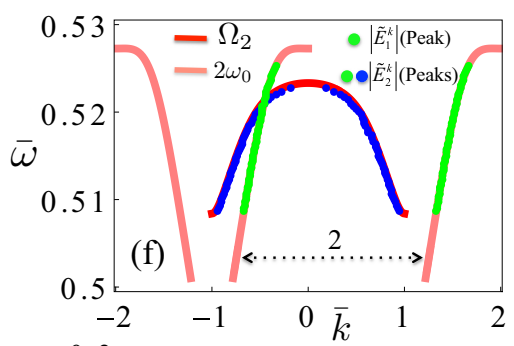

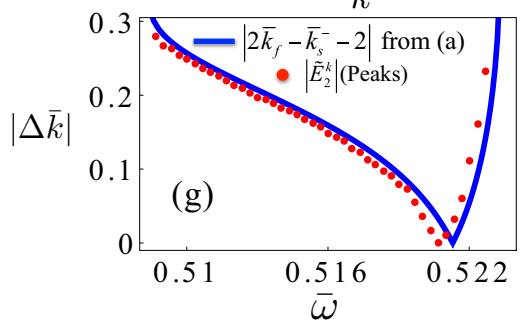

FIG. 4. SHG via double valley-Hall kink states. (a) Dispersion curves (left) used for the SHG [from the bottom panel of Fig. 3(b) at $r_{1}=0.19 a, r_{2}=0.24 a$, where the shaded dark gray area is occupied by the bulk modes, whereas the light blue (light gray) indicates the frequency-matching window and chirality maps (right) of the valley modes at $k_{f}$ and $k_{s}^{+}$. (b) Simulated field intensities of the fundamental ( $E_{1}$ ) and second-harmonic $\left(E_{2}\right)$ waves at the frequency marked by the blue (dark gray) line in (a). The fundamental wave is excited unidirectionally via a chiral source located at the extremum of the $k_{f}$ chirality map, which is realized by six dipoles with phase winding in the simulations. (c) and (d) Fourier transform of fields $E_{1}$ and $E_{2}$ in (b) (both are normalized by the maximum of $\left|\tilde{E}_{1}^{k}\right|$ ). (e) The forward-to-backward ratio $\eta_{\rightleftarrows}$ of the generated second-harmonic waves vs frequency determined from the amplitudes of the peaks at $\bar{k}_{s}^{-}$and $\bar{k}_{s}^{+}$in (d). (f) Extracted dispersion curves from the peaks of $\left|\tilde{E}_{1}^{k}\right|$ and $\left|\tilde{E}_{2}^{k}\right|$ [green (medium gray) dots for the fundamental wave and blue (dark gray) dots for the harmonic wave) in (c) and (d) compared to the dispersion curves of (a). (g) Extracted $|\Delta \bar{k}|$ from the peaks of $\left|\tilde{E}_{2}^{k}\right|$ in (d) compared to that obtained from the dispersion curves of (a).

excellent agreement demonstrates that the SHG observed in the full-wave simulations is, indeed, due to the nonlinear coupling of the kink modes located in the two valley gaps. Figure 4(g) further shows the extracted wave-vector mismatch $\Delta \bar{k}$ from the peaks of $\left|\tilde{E}_{2}^{k}\right|$ compared to that obtained from the eigenmode dispersion curves in Fig. 4(a). One can again see good agreement considering possible finite-size effects and different algorithms used in the full-wave simulation and the eigenmode calculation. Last, but not least, we would also like to note that the valley-Hall kink modes are robust against structural disorder [48].

Application to enhanced enantioselectivity in probing chiral molecules. Our system exhibits several features that could
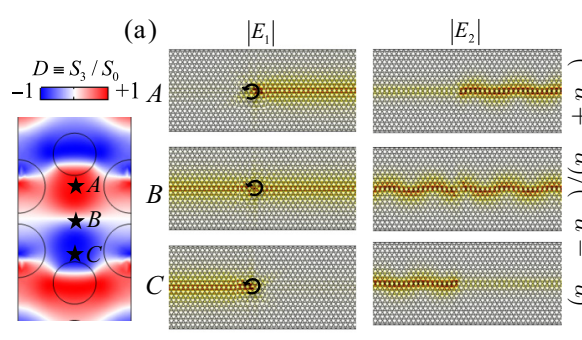

(b)

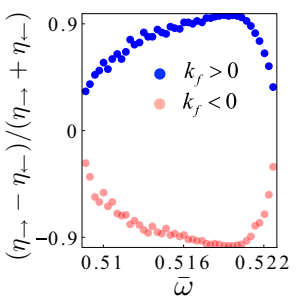

FIG. 5. Direction-tunable SHG and nonlinear directional dichroism. (a) The propagation direction of the SHG could be switched from rightward to leftward by changing the source location from $A$ to $C$ as labeled in the chirality map. (b) The SHG-DD, defined as $\left(\eta_{\rightarrow}-\eta_{\leftarrow}\right) /\left(\eta_{\rightarrow}+\eta_{\leftarrow}\right)$, determined for $k_{f}>0\left(k_{f}<0\right)$, corresponding to case $A(C)$ in (a). lead to active devices with different functionalities. For example, the chirality map suggests that one could tune the direction of the SHG by simply changing the source location of the fundamental wave (location of chiral molecules), as demonstrated in Fig. 5(a). Importantly, the plots in Fig. 5(b) show that using our proposed device, one can achieve remarkably large $S H G$ directional dichroism (SHG-DD), defined as $\left(\eta_{\rightarrow}-\eta_{\leftarrow}\right) /\left(\eta_{\rightarrow}+\eta_{\leftarrow}\right)$. In particular, the maximum value of the SHG-DD, achieved for $\bar{\omega}=0.52$, is as large as 0.97 . This is much larger than what can be achieved with optical systems employing superchiral light [49-51] or comparatively much bulkier nonlinear metasurfaces [52-54] (see [45] for a detailed quantitative characterization of such optical probes for sensing chiral molecules).

Conclusion and outlook. In conclusion, we have demonstrated tunable bidirectional SHG via nonlinear interaction of topological valley-Hall kink modes within two valley gaps in all-dielectric $\mathrm{PhC}$ structures. The ideas presented here could be extended to other nonlinear optical processes, e.g., third-harmonic generation or four-wave mixing. Implementing nonlinear frequency mixing processes using quantum spin Hall edge modes [17] could also lead to new physics. Moreover, one could explore other platforms, such as waveguide arrays [55] and coupled resonators [13], to achieve nonlinear topological physics similar to that observed in the current setup.

Acknowledgments. This work was supported by the European Research Council (ERC; Grant No. ERC-2014CoG-648328). W.E.I.S. acknowledges support from a Marie Sklodowska-Curie Individual Fellowship (MSCA-IFEF-ST752898). 
[1] L. Lu, J. D. Joannopoulos, and M. Soljacic, Nat. Photonics 8, 821 (2014).

[2] A. B. Khanikaev and G. Shvets, Nat. Photonics 11, 763 (2017).

[3] X.-C. Suna, C. He, X.-P. Liu, M.-H. Lu, S.-N. Zhu, and Y.-F. Chen, Prog. Quantum Electron. 55, 52 (2017).

[4] Y. Wu, C. Li, X. Hu, Y. Ao, Y. Zhao, and Q. Gong, Adv. Opt. Mater. 5, 1700357 (2017).

[5] B.-Y. Xie, H.-F. Wang, X.-Y. Zhu, M.-H. Lu, Z. D. Wang, and Y.-F. Chen, Opt. Express 26, 24531 (2018).

[6] M. S. Rider, S. J. Palmer, S. R. Pocock, X. Xiao, P. A. Huidobro, and V. Giannini, J. Appl. Phys. 125, 120901 (2019).

[7] T. Ozawa, H. M. Price, A. Amo, N. Goldman, M. Hafezi, L. Lu, M. C. Rechtsman, D. Schuster, J. Simon, O. Zilberberg, and I. Carusotto, Rev. Mod. Phys. 91, 015006 (2019).

[8] F. D. M. Haldane and S. Raghu, Phys. Rev. Lett. 100, 013904 (2008).

[9] Z. Wang, Y. D. Chong, J. D. Joannopoulos, and M. Soljacic, Phys. Rev. Lett. 100, 013905 (2008).

[10] Z. Wang, Y. D. Chong, J. D. Joannopoulos, and M. Soljacic, Nature (London) 461, 772 (2009).

[11] Y. Poo, R.-X. Wu, Z. Lin, Y. Yang, and C. T. Chan, Phys. Rev. Lett. 106, 093903 (2011).

[12] M. C. Rechtsman, J. M. Zeuner, Y. Plotnik, Y. Lumer, D. Podolsky, F. Dreisow, S. Nolte, M. Segev, and A. Szameit, Nature (London) 496, 196 (2013).

[13] M. Hafezi, S. Mittal, J. Fan, A. Migdall, and J. M. Taylor, Nat. Photonics 7, 1001 (2013).

[14] A. B. Khanikaev, S. H. Mousavi, W. K. Tse, M. Kargarian, A. H. MacDonald, and G. Shvets, Nat. Mater. 12, 233 (2013).

[15] T. Ma, A. B. Khanikaev, S. H. Mousavi, and G. Shvets, Phys. Rev. Lett. 114, 127401 (2015).

[16] C. He, X.-C. Sun, X.-P. Liu, M.-H. Lu, Y. Chen, L. Feng, and Y.-F. Chen, Proc. Natl. Acad. Sci. USA 113, 4924 (2016).

[17] L. H. Wu and X. Hu, Phys. Rev. Lett. 114, 223901 (2015).

[18] T. Ma and G. Shvets, New J. Phys. 18, 025012 (2016).

[19] X. T. He, E. T. Liang, J. J. Yuan, H. Y. Qiu, X. D. Chen, F. L. Zhao, and J. W. Dong, Nat. Commun. 10, 872 (2019).

[20] M. I. Shalaev, W. Walasik, A. Tsukernik, Y. Xu, and N. M. Litchinitser, Nat. Nanotechnol. 14, 31 (2019).

[21] J. Ma, X. Xi, and X. Sun, Laser Photonics Rev. 13, 1900087 (2019).

[22] S. Rachel, Rep. Prog. Phys. 81, 116501 (2018).

[23] R. W. Boyd, Nonlinear Optics (Elsevier, New York, 2008).

[24] D. Smirnova, D. Leykam, Y. Chong, and Y. Kivshar, Appl. Phys. Rev. 7, 021306 (2020).

[25] Y. Lumer, Y. Plotnik, M. C. Rechtsman, and M. Segev, Phys. Rev. Lett. 111, 243905 (2013).

[26] D. Leykam and Y. D. Chong, Phys. Rev. Lett. 117, 143901 (2016).

[27] S. Mukherjee and M. C. Rechtsman, Science 368, 856 (2020).

[28] D. A. Dobrykh, A. V. Yulin, A. P. Slobozhanyuk, A. N. Poddubny, and Y. S. Kivshar, Phys. Rev. Lett. 121, 163901 (2018).

[29] D. Smirnova, S. Kruk, D. Leykam, E. Melik-Gaykazyan, D.-Y. Choi, and Y. Kivshar, Phys. Rev. Lett. 123, 103901 (2019).

[30] V. Peano, M. Houde, F. Marquardt, and A. A. Clerk, Phys. Rev. X 6, 041026 (2016)
[31] G. Harari, M. A. Bandres, Y. Lumer, M. C. Rechtsman, Y. D. Chong, M. Khajavikhan, D. N. Christodoulides, and M. Segev, Science 359, eaar4003 (2018).

[32] M. A. Bandres, S. Wittek, G. Harari, M. Parto, J. Ren, M. Segev, D. N. Christodoulides, and M. Khajavikhan, Science 359, eaar4005 (2018).

[33] S. Mittal, E. A. Goldschmidt, and M. Hafezi, Nature (London) 561, 502 (2018).

[34] C. Qian, K. H. Choi, R. P. H. Wu, Y. Zhang, K. Guo, and K. H. Fung, Opt. Express 26, 5083 (2018).

[35] Y. Wang, L.-J. Lang, C. H. Lee, B. Zhang, and Y. D. Chong, Nat. Commun. 10, 1102 (2019).

[36] S. Kruk, A. Poddubny, D. Smirnova, L. Wang, A. Slobozhanyuk, A. Shorokhov, I. Kravchenko, B. Luther-Davies, and Y. S. Kivshar, Nat. Nanotechnol. 14, 126 (2019).

[37] J. W. You, Z. Lan, and N. C. Panoiu, Sci. Adv. 6, eaaz3910 (2020).

[38] Z. Lan, J. W. You, and N. C. Panoiu, Phys. Rev. B 101, 155422 (2020).

[39] X.-D. Chen, F.-L. Zhao, M. Chen, and J.-W. Dong, Phys. Rev. B 96, 020202(R) (2017).

[40] Y. Yang, H. Jiang, and Z. H. Hang, Sci. Rep. 8, 588 (2018).

[41] BANDSOLVE, https://www.synopsys.com.

[42] R. Zhao, G.-D. Xie, M. L. N. Chen, Z. Lan, Z. Huang, and W. E. I. Sha, Opt. Express 28, 4638 (2020).

[43] C. Wang, H. Zhang, H. Yuan, J. Zhong, and C. Lu, Front. Optoelectron. 13, 73 (2020).

[44] M. B. de Paz, C. Devescovi, G. Giedke, J. J. Saenz, M. G. Vergniory, B. Bradlyn, D. Bercioux, and A. G. Etxarri, Adv. Quantum Technol. 3, 1900117 (2020).

[45] See Supplemental Material at http://link.aps.org/supplemental/ 10.1103/PhysRevA.103.L041502 for details of topological properties and chirality map of the valley-Hall kink modes, direction-tunable SHG via varying source location and forwardto-backward directional dichroism.

[46] Y. Wang, J. W. You, Z. Lan, and N. C. Panoiu, Opt. Lett. 45, 3151 (2020).

[47] J. W. You, Z. Lan, Q. Bao, and N. C. Panoiu, IEEE J. Sel. Top. Quantum Electron. 26, 4600308 (2020).

[48] Z. Dong, F. Xu, and W. Liang, Europhys. Lett. 131, 54002 (2020).

[49] Y. Tang and A. E. Cohen, Phys. Rev. Lett. 104, 163901 (2010).

[50] Y. Tang and A. E. Cohen, Science 332, 333 (2011).

[51] M. Schaferling, D. Dregely, M. Hentschel, and H. Giessen, Phys. Rev. X 2, 031010 (2012).

[52] V. K. Valev, J. J. Baumberg, B. De Clercq, N. Braz, X. Zheng, E. J. Osley, S. Vandendriessche, M. Hojeij, C. Blejean, J. Mertens, C. G. Biris, V. Volskiy, M. Ameloot, Y. Ekinci, G. A. E. Vandenbosch, P. A. Warburton, V. V. Moshchalkov, N. C. Panoiu, and T. Verbiest, Adv. Mater. 26, 4074 (2014).

[53] S. Chen, F. Zeuner, M. Weismann, B. Reineke, G. Li, V. K. Valev, K. W. Cheah, N. C. Panoiu, T. Zentgraf, and S. Zhang, Adv. Mater. 28, 2992 (2016).

[54] D. Kim, J. Yu, I. Hwang, S. Park, F. Demmerle, G. Boehm, M. C. Amann, M. A. Belkin, and J. Lee, Nano Lett. 20, 8032 (2020).

[55] J. Noh, S. Huang, K. P. Chen, and M. C. Rechtsman, Phys. Rev. Lett. 120, 063902 (2018). 